\title{
Study on fruit quality measurement and evaluation based on color identification
}

\author{
Yizhong Wang *a ${ }^{\text {, }}$ Yanhua Cui ${ }^{\mathrm{a}}$, Shaohui Chen ${ }^{\mathrm{b}}$, Ping Zhang ${ }^{\mathrm{b}}$, Huafang Huang ${ }^{\mathrm{a}}$, George Q. Huang ${ }^{\mathrm{c}}$ \\ ${ }^{\mathrm{a}}$ School of Electronic Information and Automation, Tianjin University of Science and Technology, \\ Tianjin 300457, P. R. China; \\ bNational Engineering and Technology Research Center for Preservation of Agricultural Products, \\ Tianjin 300384, P. R. China \\ ${ }^{\mathrm{c}}$ Department of Industrial and Manufacturing Systems Engineering, The University of Hong Kong, \\ Hong Kong, P. R. China
}

\begin{abstract}
A non-destructive measuring and evaluating method for fruits is proposed based on color identification. The color images of fruits are taken firstly. Then, images' RGB histograms are calculated and used as quality parameters for fruits. A BP neural network with three layers is established. Its input and output are the RGB histograms and evaluating results, respectively. After training, the qualities of fruits are identified by the BP network according to the histogram of RGB of fruits' images. For verifying the proposed method, the qualities of bananas are measured and evaluated. Experiment results show the reliability and feasibility of proposed method.
\end{abstract}

Keywords: Color identification, fruit, quality evaluation, BP neural network, RGB color model

\section{INTRODUCTION}

Quality measurement and evaluation have been one of the important issues in agricultural product logistics. In the storage, transportation and sale of fruits, quality measurement, assessment and classification are needed. Surface color is one of characters to represent the quality status of some fruits. As it contains basic image information, color is one of the most important image features. Also, color is the elementary information stored in pixels.

In this paper, in order to measure and evaluate the quality of fruits non-destructively, their color images are taken. The images are presented with RGB color model, which is the most commonly used color model. The RGB color model is an additive color model ${ }^{1}$. Different colors can be produced by adding red, green, and blue light together in various ways. The RGB color model is used in this paper for measuring and evaluating the quality of fruit.

To describe the colors of fruits' images, the histograms of RGB are used. To identify the color of fruits' images, a BP neural network is designed. The histograms of RGB are input the BP network, and the output if the level of fruit quality evaluation.

\section{RGB COLOR MODEL AND COLOR HISTOGRAMS}

\subsection{RGB color model}

When a color image of fruit is taken by a camera, the image is in the RGB color model. The RGB color model is an additive model in which red, green, and blue (often used in additive light models) are combined in various ways to reproduce other colors. The name of the model and the abbreviation 'RGB' comes from the three primary colors, red, green, and blue. In a general sense, the RGB color model describes our perception of color. Three types of receptors in the retina of the human eye have peak sensitivities corresponding to these three primary colors. As shown in Fig.1, the RGB color model represents colors within a cubic volume defined by orthogonal Red, Green, and Blue axes ${ }^{2}$. Black is at the origin of the coordinate system $(\mathrm{R}=\mathrm{G}=\mathrm{B}=0)$, and white is at the opposite corner of the cube $(\mathrm{R}=\mathrm{G}=\mathrm{B}=255)$. The diagonal connecting the black and white corners (dashed line) contains the range of neutral gray levels.

*yzwang@tust.edu.cn; phone 86-22-60272649; fax 86-22-60274490

2009 International Conference on Optical Instruments and Technology: Optoelectronic Imaging and Process Technology, edited by Toru Yoshizawa, Ping Wei, Jesse Zheng, Proc. of SPIE

Vol. 7513, 75130F · ( 2009 SPIE · CCC code: 0277-786X/09/\$18 · doi: 10.1117/12.839698

Proc. of SPIE Vol. $751375130 \mathrm{~F}-1$ 


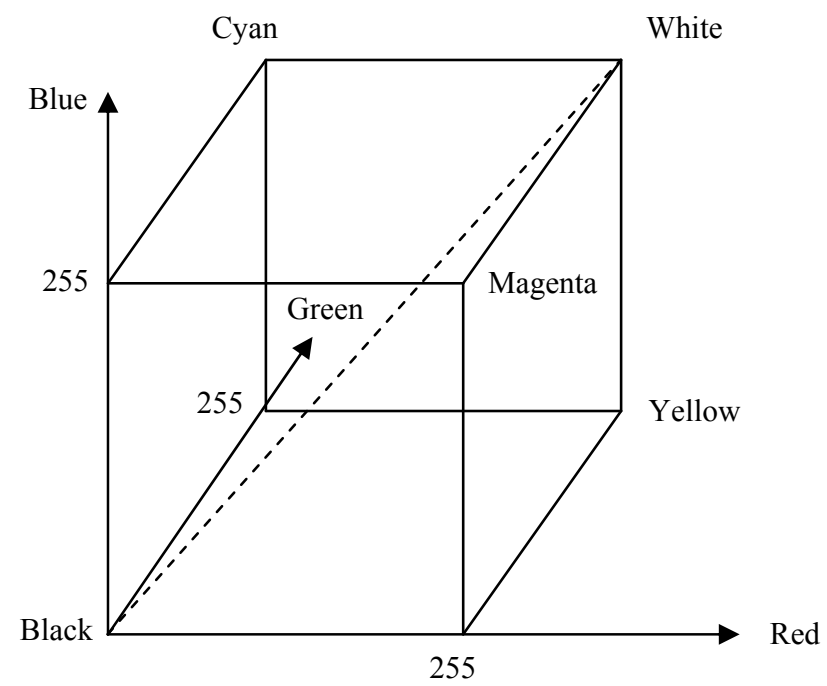

Fig. 1 RGB color model

\subsection{Color histograms}

A histogram is a graphical display of tabulated frequencies, shown as bars. It shows what proportion of cases fall into each of several categories. In image processing, a color histogram is a representation of the distribution of colors in an image. It is derived by counting the number of pixels of each given set of color ranges in a color space. Color histograms are useful tool in image processing. The color histogram is defined as ${ }^{3}$ :

$$
h_{A, B, C}\left[r_{1}, r_{2}, r_{3}\right]=N \bullet P\left\{A=r_{1}, B=r_{2}, C=r_{3}\right\}
$$

where $A, B$, and $C$ represent the three color channels $(R, G, B) ; N$ is the total number of pixels in the image; $P$ is probability; $r_{1}, r_{2}, r_{3}$ are the color values. After the color of an image is quantitated, color histograms can be obtained by calculating the frequencies of every color's appearance. As an example, the RGB histograms of the color image of a banana, shown in Fig. 2, are given in Fig. 3.

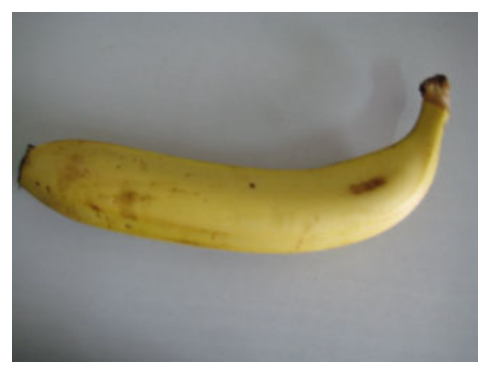

Fig. 2 Image of a banana 


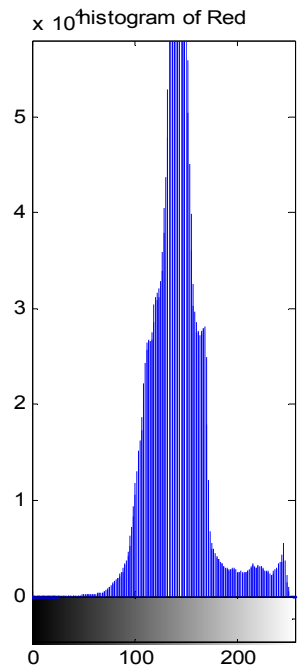

(a) Histogram of $\mathrm{R}$

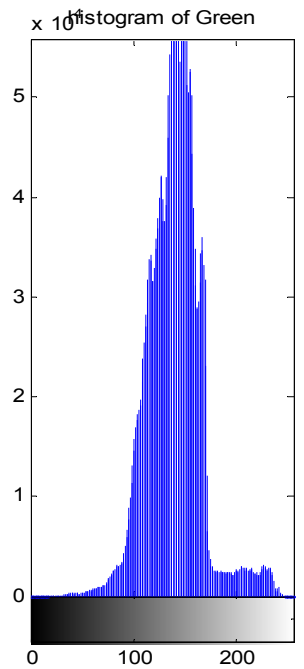

(b) Histogram of G

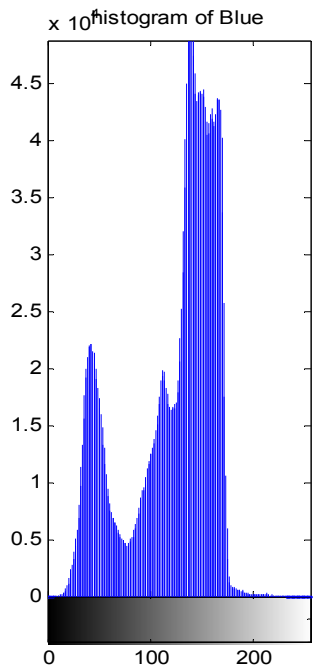

(c) Histogram of B

Fig. 3 Color histograms of the banana image

\section{QUALITY MEASUREMENT AND EVALUATION OF FRUIT}

\subsection{BP neural network}

The error back propagation neural network, which is referred to as BP artificial neural network, is more mature in the forecast at present and the most widely used artificial neural network technology ${ }^{4-5}$. It is a multi-layer architecture, with one input layer, one output layer, and one or more intermediate layers. Each node within the network represents a neuron. The neurons connect neurons in other layer, not in the same layer. Each neuron sums weighted inputs, and then applies a linear or nonlinear function to the resulting sum to determine the output.

BP learning algorithm is a supervised learning algorithm. Supervised-learning is applied for the most common neural network training procedure ${ }^{6}$. In the supervised-learning process, the neural network is presented with an input pattern together with the target output for that pattern. The target output is supposed to be the correct corresponding outcome for the input pattern. BP neural network has great self-study and self-organize ability ${ }^{7}$. It can accurately establish the mapping between the input and output variables, and can approximate an arbitrary nonlinear function with better precision.

\subsection{Fruit quality measurement and evaluation}

In the storage, transportation and sale of fruits, the surface colors of some fruits, such as banana, represent their quality change and status. For indentifying the color of the image of a fruit with a BP network, there will be a lot of neurons in the input layer if the colors histograms shown in Fig. 3 are used. In this paper, simplified color histograms, illustrated in Fig. 4, are used. Each color is divided into five levels. Therefore, there are totally 15 neurons in the input layer of the designed BP network. The quality of fruit is also divided into five levels. Binary values are used for the output of the BP network. The quality level values, namely $9,7,5,3$, and 1 from high to low, correspond with $\left(\begin{array}{llllllll}1 & 0 & 0 & 0 & 0\end{array}\right),\left(\begin{array}{lllll}0 & 1 & 0 & 0 & 0\end{array}\right),\left(\begin{array}{lll}0 & 0\end{array}\right.$

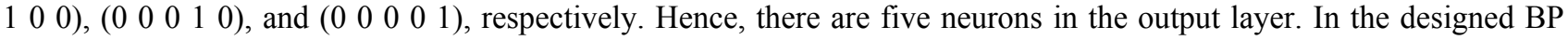
network, depicted in Fig. 5, there are 31 neurons in the intermediate layer. 


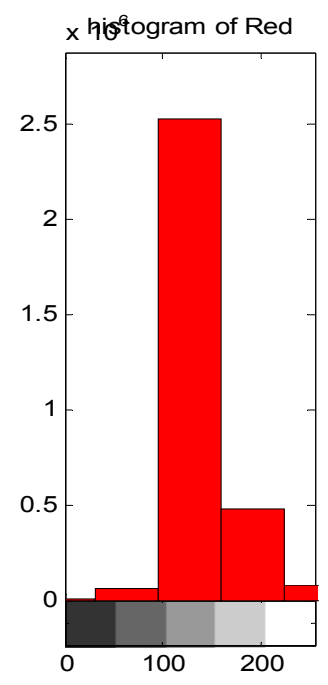

(a) Histogram of $\mathrm{R}$

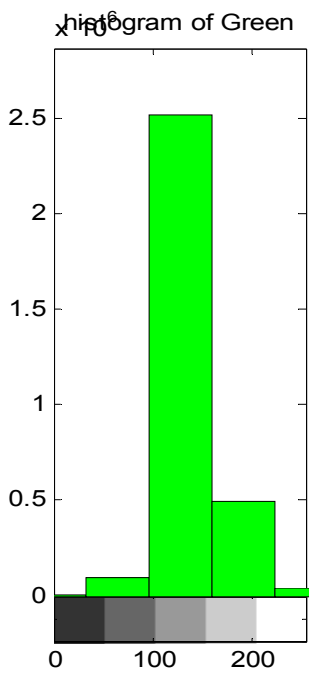

(b) Histogram of G

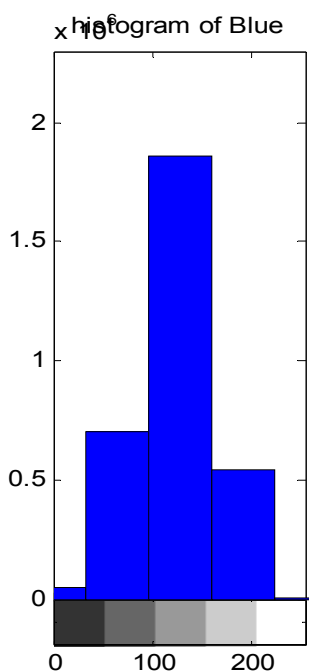

(c) Histogram of B

Fig. 4 Simplified color histograms

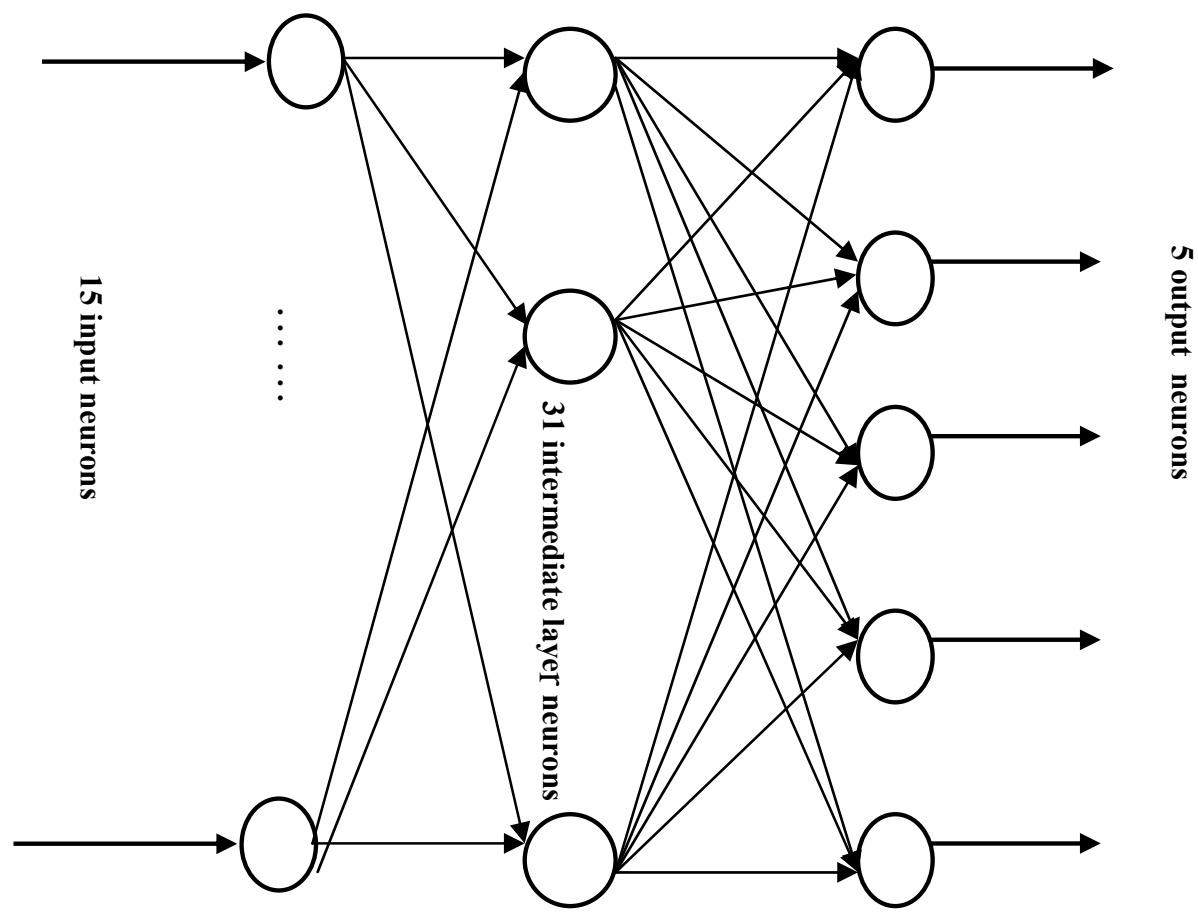

Fig. 5 Designed BP neural network

\section{CASE STUDY}

To verify the proposed method, experiments of banana quality measurement and evaluation are conducted. Ten banana images at different status, given in Fig. 6, are used to train the designed BP network. Then, the trained BP network is used to measure and evaluate the banana images in Fig. 7. Their quality values are 9, 5, and 3, respectively, being consistent with their qualities. 


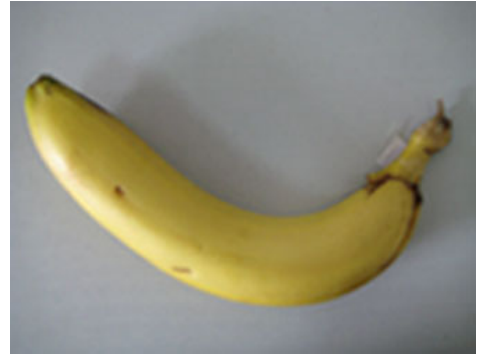

(a) Sample 1

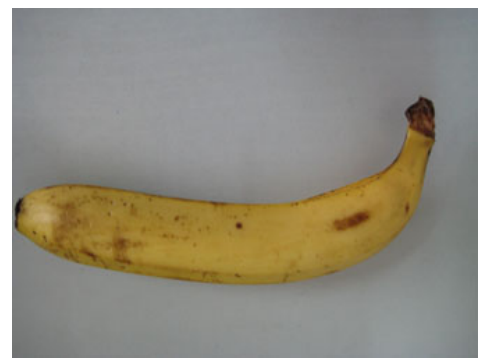

(d) Sample 4

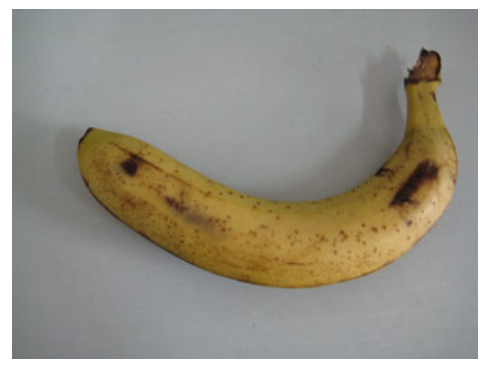

(g) Sample 7

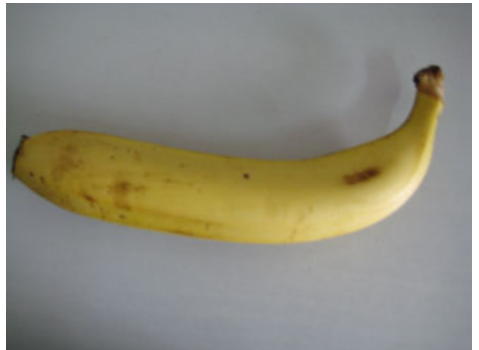

(b) Sample 2

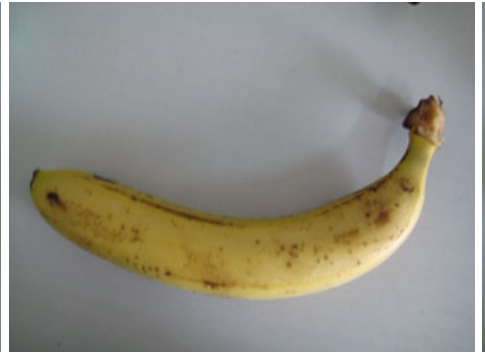

(e) Sample 5

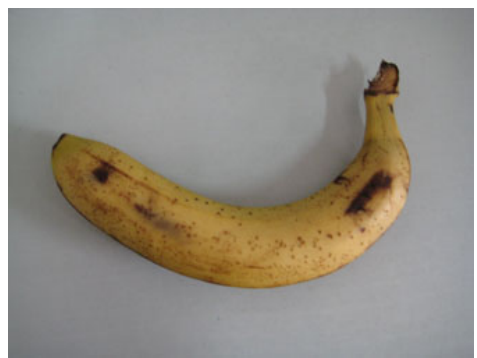

(h) Sample 8

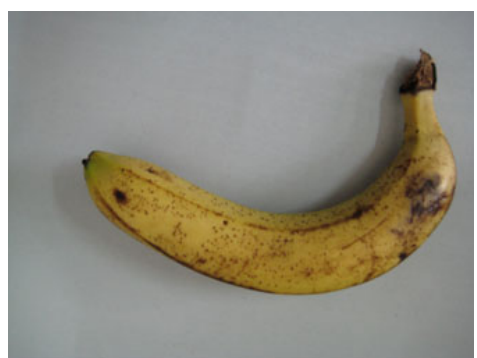

(j) Sample 10

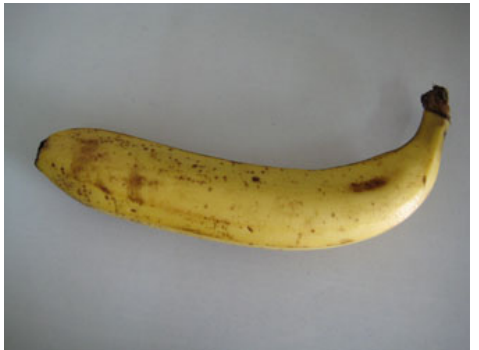

(c) Sample 3

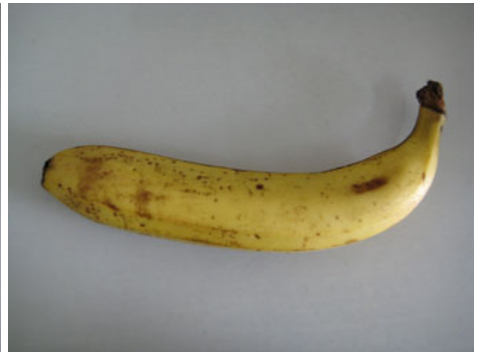

(f) Sample 6

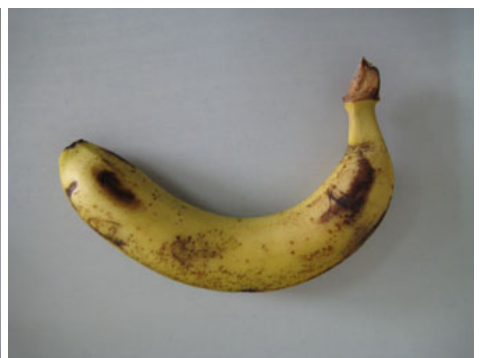

(i) Sample 9

Fig. 6 Banana images for training the BP network 


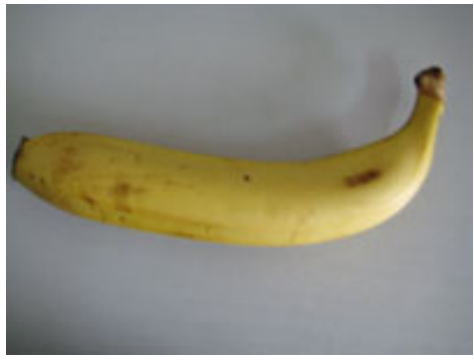

(a) Test sample 1

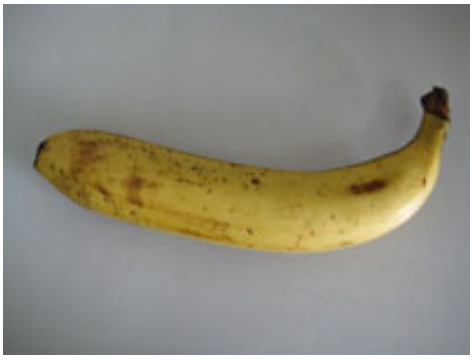

(b) Test sample 2

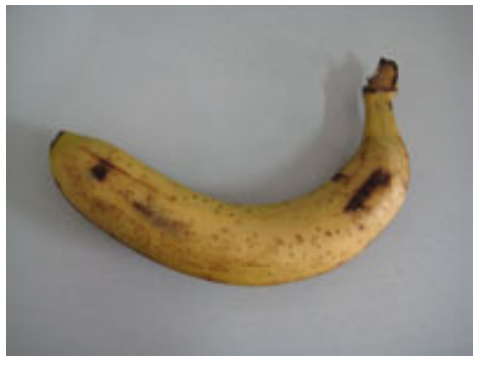

(c) Test sample 3

Fig. 7 Banana images for testing the BP network

\section{CONCLUSION}

In this paper, a measuring and evaluating method for fruit quality is proposed using the surface color as an evaluation parameter. A BP neural network with three layers is designed for this. Simplified color histograms of fruit image are used as the input of the BP network. And the evaluation results are the output. To verify the proposed method, measurement and evaluation experiments of bananas are conducted. Experiment results shows the feasibility and usefulness of the proposed method.

\section{ACKNOWLEDGMENTS}

This research is carried out with a grant from Tianjin Municipal Science and Technology Commission under the code 08ZCKFNC00700.

\section{REFERENCES}

[1] Gonzalez, R. C. and Woods, R. E., [Digital image processing], Prentice Hall, N.J., 290-301 (2002).

[2] Fu, B. D., Yuan, J. C., and Guo, C. X., "Distinguished arithmetic for cotton impurity based on RGB color model," Beijing Textile Journal, 26(5), 48-51 (2005).

[3] He, D. J., [The digital image processing], Xidian University Press, Xi’an, 210 (2008).

[4] He, L. Q. [The artificial neural network theory, design and application research], Chemical Industry Press, Beijing, 43-46 (2002).

[5] Hagan, M. T., Demuth, H. B., and Beale M., [Neural network design], PWS Pub., Boston, Mass., (1996).

[6] Kondo, N., Ahmad, U, Monta, M, and Murase, H., "Machine vision based quality evaluation of Iyokan orange fruit using neural networks," Computers and Electronics in Agriculture, 29(1-2), 135-147 (2000).

[7] Dayhoff, J. E., [Neural network architectures: an introduction], Van Nostrand Reinhold, New York, (1990). 